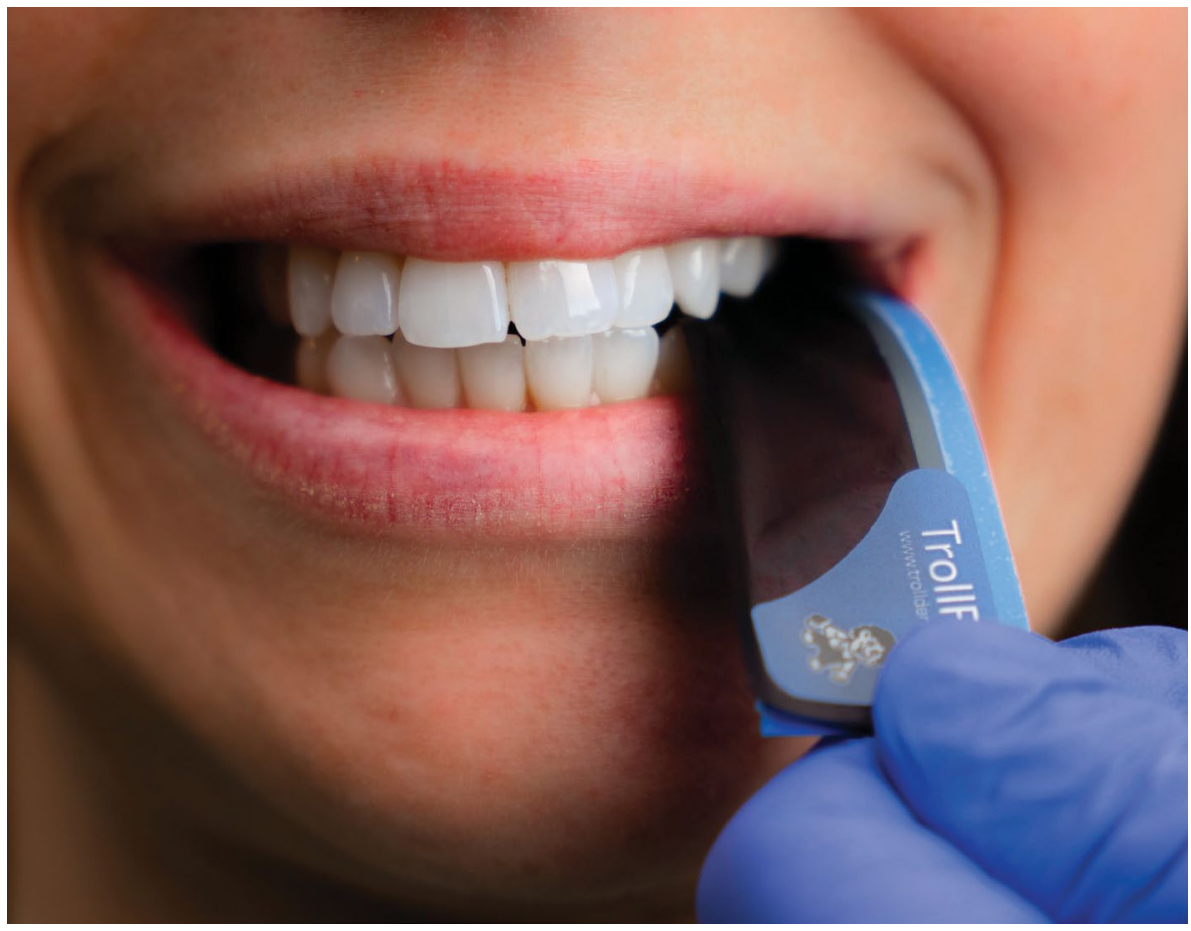

Occlusal adjustment is usually a timeconsuming process and a very critical phase in restorative dentistry, however most dentists do not dedicate adequate attention to this process. Research shows that around $95 \%$ of occlusal readings are incorrect. Misreading urges the dentist to remove tissues from occlusal spots which can lead to occlusal disturbance.

In crown delivery, any undetected high occlusal point can lead to a range of consequences such as minimal cracks in the crown, to TMJ pain and sometimes to failure of the implant in case of crown over implant. In some situations, minor errors can be self-adjusted, but this is not always the case.

Despite the introduction of computerised occlusal analysis devices, articulating paper remains the most common means to check occlusion. The sensitivity and reliability of the techniques applied for occlusal analysis when using articulating paper depends on its thickness, composition, ink substrate and plastic deformation. The oral environment (saliva, humidity) also plays an important role. Another factor that affects the readings when using articulating paper is the restorative material used. Conventionally, the material used in fixed and removable prosthodontics were either metal, composite, porcelain fused to metal, veneered zirconia or acrylic resin. The recent introduction of full contour zirconia, either manually polished or furnace glazed, can affect the interpretation of the reading of the articulating paper.

To meet these challenges, the Swedish occlusion foil TrollFoil (Directa) is the best ally of any dentist. This extremely precise, double-sided articulation foil can be used under a wide variety of clinical situations including wet or dry teeth, limited opening, limited vestibular space, gaggers, on restorations in any material including highly polished surfaces such as zirconia and e.max.

TrollFoil is mounted on a rigid plastic frame that keeps it stretched and straight, enabling the operator to hold it without the help of forceps.

Research shows that the thinner the articulating paper, the more precise marks you get. Thick articulating paper generates a less uniform and larger contact area than the occlusal area marked with thin articulating foil. This is why TrollFoil measures only 8 micron and is now also available in a new 4.5 micron version - the thinnest available foil. It proves especially useful when checking the occlusion on implants that have a very smooth and shiny surface. For information contact info@ directadental.com.

\title{
Safe and precise implant surgery
}

Did you know W\&H offers a handpiece specifically designed to support Zygomatic dental implant treatment?

Developed in collaboration with leaders from the field, the new SZ-75 handpiece features a unique angulation that enables optimal access to the treatment site. It also boasts an innovative chuck system for improved safety and highly accurate concentricity during application.

The SZ-75 can be combined with W\&H's award-winning Implantmed surgical unit, which facilitates atraumatic and precise implant placement with its automatic
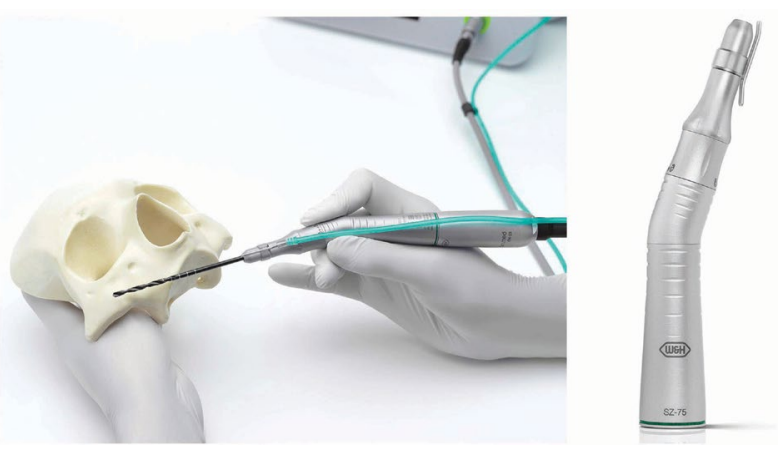

thread cutter and torque control function.

The Implantmed's wireless foot control also enables you to operate the unit from your ideal working position.
Call now for further details.

To find out more visit www.wh.com/ en_uk, call 01727874990 or email office. uk@wh.com. 